Special Communication

\title{
The European cross-border health data exchange roadmap: Case study in the Italian setting
}

\author{
Marco Nalin $^{\mathrm{a}}$, Ilaria Baroni ${ }^{\mathrm{a}}$, Giuliana Faiella ${ }^{\mathrm{b}, *}$, Maria Romano $^{\mathrm{a}}$, Flavia Matrisciano ${ }^{\mathrm{b}}$, \\ Erol Gelenbe ${ }^{\mathrm{c}}$, David Mari Martinez ${ }^{\mathrm{d}}$, Jos Dumortier ${ }^{\mathrm{e}}$, Pantelis Natsiavas ${ }^{\mathrm{f}}$, Kostas Votis ${ }^{g}$, \\ Vassilis Koutkias ${ }^{\mathrm{f}}$, Dimitrios Tzovaras ${ }^{\mathrm{g}}$, Fabrizio Clemente ${ }^{\mathrm{h}}$ \\ ${ }^{a}$ Telbios S.r.l, Milan, Italy \\ ${ }^{\mathrm{b}}$ Fondazione Santobono Pausilipon, Naples, Italy \\ ${ }^{\mathrm{c}}$ Department of Electrical and Electronic Engineering, Imperial College of Science, Technology and Medicine, London, UK \\ ${ }^{\mathrm{d}}$ Eurecat - Centro Tecnológico de Catalunya, Barcelona, Spain \\ e Time.lex, Brussels, Belgium \\ ${ }_{\mathrm{f}}^{\mathrm{f}}$ Institute of Applied Biosciences, Centre for Research \& Technology Hellas, Thermi, Thessaloniki, Greece \\ ${ }^{\mathrm{g}}$ Information Technologies Institute, Centre for Research \& Technology Hellas, Thermi, Thessaloniki, Greece \\ ${ }^{\mathrm{h}}$ Institute of Cristallography - National Research Center (CNR), Rome, Italy
}

\section{A R T I C L E I N F O}

\section{Keywords:}

Cross-border health data exchange

Interoperability

Ethics

Regulatory issues

Cybersecurity

\begin{abstract}
A B S T R A C T
Health data exchange is a major challenge due to the sensitive information and the privacy issues entailed. Considering the European context, in which health data must be exchanged between different European Union (EU) Member States, each having a different national regulatory framework as well as different national healthcare structures, the challenge appears even greater. Europe has tried to address this challenge via the epSOS ("Smart Open Services for European Patients") project in 2008, a European large-scale pilot on crossborder sharing of specific health data and services. The adoption of the framework is an ongoing activity, with most Member States planning its implementation by 2020. Yet, this framework is quite generic and leaves a wide space to each EU Member State regarding the definition of roles, processes, workflows and especially the specific integration with the National Infrastructures for eHealth. The aim of this paper is to present the current landscape of the evolving eHealth infrastructure for cross-border health data exchange in Europe, as a result of past and ongoing initiatives, and illustrate challenges, open issues and limitations through a specific case study describing how Italy is approaching its adoption and accommodates the identified barriers. To this end, the paper discusses ethical, regulatory and organizational issues, also focusing on technical aspects, such as interoperability and cybersecurity. Regarding cybersecurity aspects per se, we present the approach of the KONFIDO EU-funded project, which aims to reinforce trust and security in European cross-border health data exchange by leveraging novel approaches and cutting-edge technologies, such as homomorphic encryption, photonic Physical Unclonable Functions (p-PUF), a Security Information and Event Management (SIEM) system, and blockchainbased auditing. In particular, we explain how KONFIDO will test its outcomes through a dedicated pilot based on a realistic scenario, in which Italy is involved in health data exchange with other European countries.
\end{abstract}

\section{Introduction}

Patient data exchange among healthcare organizations is a significant challenge due to the sensitive information and the privacy issues involved. In Europe, the right of a patient for cross-border healthcare is defined in Directive 2011/24/EU of the European Parliament and of the Council of 9 March 2011 [1]. However, in practical terms cross-border healthcare is quite complicated, since each
European country has its own national regulatory framework as well as its National Healthcare System infrastructures/organizations/roles, etc., hampering efficient health data exchange.

Thus, this paper outlines the current landscape regarding the establishment and deployment of an interoperable and secure crossborder health data exchange framework in Europe. First, it outlines the strategy that the Europe Union (EU) has undertaken since 2008 to build a framework for interoperable exchange of eHealth information within

\footnotetext{
* Corresponding author.

E-mail address: giuliana.faiella@gmail.com (G. Faiella).
} 
EU Member States, starting with Patient Summary and the ePrescription services, through the epSOS (Smart Open Services for European Patients) initiative [2].

epSOS and OpenNCP (the software implementation of epSOS [2]) are developed and tested by European projects such as eStandards, Trillium-II and International Patient Summary project (IPS). On this basis, the European Commission commissioned the European Committee for standardization (CEN) to create the European Standard 17269 titled 'The Patient Summary for Unplanned, Cross-border Care' [3]. Despite establishing a basic, common framework for EU Member States, the adoption of the standard by the different Member States requires time and effort and therefore several key barriers are identified. Furthermore, the paper presents the adoption of epSOS in Italy, a Member State that is particularly interesting because of its federated healthcare system, where the different Italian regions are the actual actors involved in the management of patients' data. Besides technical aspects, the paper discusses important challenges concerning, legal, organizational and ethical issues, which are also applicable in this context. Since numerous actors are involved in the exchange of personal health data, important cybersecurity threats may arise with societal and ethical implications. To this end, the paper introduces the approach followed by the KONFIDO ("Secure and Trusted Paradigm for Interoperable eHealth Services") EU-funded project [4,5], which develops tools and procedures to reinforce the security of cross-border health data exchange across EU Member States. In particular, KONFIDO leverages novel approaches and cutting-edge technologies, such as homomorphic encryption, photonic Physical Unclonable Functions (pPUF), a Security Information and Event Management (SIEM) system, and blockchain-based auditing [6]. In order to validate its innovative toolset, KONFIDO plans a pilot based on the Italian National infrastructure, taking into account the major regulatory, ethical and technical barriers identified.

\section{Background}

In 2008 the epSOS initiative was launched in Europe, involving initially a few stakeholders, but gradually expanded to a large-scale pilot encompassing 25 countries and 50 beneficiaries. The initiative was partially founded by the Information \& Communication Technology Policy Support Programme (ICT PSP), as part of the Competitiveness and Framework Programme by the European Commission, and it was completed in 2014. The main goal of epSOS was to develop a practical eHealth framework and an Information \& Communication Technology infrastructure for enabling interoperable access to patient health information, with respect to basic documents namely Patient Summary (PS) and ePrescription (eP) between different EU healthcare systems. In particular:

- The PS is a standardized set of patient data including:

o General information about the patient (name, birth date, gender, etc.);

o A medical summary consisting of the most important patient data (e.g., allergies, current medical problems, etc.);

o A list of current medication including all prescribed medication that the patient is currently taking;

o Information about the PS itself (e.g., when and by whom was the PS generated or updated).

- The eP includes two main processes:

o the electronic prescription of drugs, transmitting the information to the pharmacy where it is being retrieved;

$o$ eDispensing (eD), i.e. the retrieval of an eP, and dispensing of the drug to the patient, and the submission of a report for the medicine dispensed.

With a special focus on interoperability, a major challenge was to map all the selected coding systems used in PS and eP into a common coding. If a country used a different coding system, a specific component needs to be deployed to convert the national coding system to the epSOS coding scheme.

The epSOS vision dictates that each country must deploy a National Contact Point for eHealth $(\mathrm{NCPeH})$, acting as a bidirectional interface between the existing national IT infrastructures and those provided by the common European infrastructures supporting eHealth functions. Besides the the semantic interpretation and translation, the NCPeH also acts as a mediator with regard to legal and regulatory aspects, as explained in the following sections. The achievements of ePSOS project have been exploited by other European projects with the aim to define a minimal yet non-exhaustive data set and the associated business rules to regulate the exchange. In order to facilitate the adoption of a common format for the Patient Summary, the European Commission urged the European Committee for standardization group of Health Informatics (CEN/TC 251) to create a standard for PS by means of the International Patient Summary (IPS) project developing also an implementation guide. The standards have been developed in partnership with Health Level-7 (HL7) using as staring point the European Guidelines on cross-border care (i.e. eHN EU PS Guidelines) that emerged from the epSOS large-scale pilot. Specifically, a draft of IPS standard, "prEN 17269 - The Patient Summary for Unplanned, Crossborder Care", was published in August 2018 and, approved in January 2019. Then, an European implementation guidance, the technical specification "prTS 17288-The International Patient Summary: Guidance for European Implementation Technical Specification", specifies how the standard PS can be deployed within a European context. These aspects will be tested in the frame of the Trillium II project that is an EU/US Cooperation for Global Interoperability in Digital Health. Trillium II is advancing the IPS standard to enable people to access and share their health information for emergency or unplanned care anywhere and as needed, including information about immunizations, allergies, medications, clinical problems, past surgical operations, and implants [3].

\section{Legal, organizational and ethical implications for cross-border in Europe}

\subsection{Legal and organizational aspects}

From a legal point of view, the healthcare providers of cross-border eHealth must comply with Directive 2011/24/EU on patients' rights in cross-border [1]. The Directive specifies the rights (i.e., the right to access data, the right to erase and correct data and the right to know who accessed data) and the rules for accessing healthcare in another EU country and one of its tasks is, precisely, to make sure that the European eHealth systems attains a high level of trust and security. A key provision of the Directive is the creation of NCPs that must have facilities to provide the information and practical assistance to patients who need to make an informed decision. For example, the NCPs should provide the credentials of the health professionals that are authorized to process healthcare data through cross-border exchange (Directive 2011/24/EU-Article 4(2)(f)). Moreover, the NCPs should provide patients with details regarding the structure of cross-border prescriptions to make it easier for patients to understand and use them. A non-exhaustive list of information that should be included in a cross-border prescription is contained in the Annex of the implementing Directive [7]. Moreover, the exchange of health data is regulated by European and national legislation regarding the protection of personal data. Currently, this domain is governed by the General Data Protection Regulation (2016/679/EC) [8].

Finally, cross-border exchange of electronic health records (EHRs) is not possible without a secure identification of patients and healthcare providers. The main legal instrument at the EU level regarding crossborder electronic identification is Regulation (EU) 2014/910, i.e. the "eIDAS Regulation", (electronic Identification, Authentication and trust 
Table 1

Legal and Organizational Requirements and Barriers.

\begin{tabular}{|c|c|}
\hline Main Legal \& Organizational Requirements & Barriers \\
\hline Patient Identification & $\begin{array}{l}\text { Not all Member States are aligned with the eIDAS Regulation and } \\
\text { the JASeHN agreement [14]. }\end{array}$ \\
\hline Patient Consent Management & $\begin{array}{l}\text { Different and complex consent mechanisms exist among Member } \\
\text { States. }\end{array}$ \\
\hline Healthcare provider authentication & $\begin{array}{l}\text { Not all Member States are aligned with the JASeHN agreement } \\
\text { [14]. }\end{array}$ \\
\hline Management of healthcare professional authorization/certification & $\begin{array}{l}\text { Not all Member States are not all aligned with JASeHN agreement } \\
\text { [14]. }\end{array}$ \\
\hline Enhance interoperability & $\begin{array}{l}\text { - Lack of EHR system among Member States. } \\
\text { Different implementation of EU regulations among Member } \\
\text { States. } \\
\text { - Different information workflows among National Infrastructure } \\
\text { and healthcare organizations. }\end{array}$ \\
\hline Accuracy and integrity of semantic processing. & $\begin{array}{l}\text { - Free text content in different languages. } \\
\text { - Lack of EHRsystem among Member States. } \\
\text { - Different technical solutions for health data digitalization. }\end{array}$ \\
\hline $\begin{array}{l}\text { Each NCPeH should be compliant with confidentiality, integrity, authenticity, availability, non-repudiation, } \\
\text { encryption, logs, audit trails, and other means of data security }\end{array}$ & $\begin{array}{l}\text { The Member States are not all aligned with the JASeHN agreement } \\
\text { [14]. }\end{array}$ \\
\hline
\end{tabular}

Services) [9]. The first part of this Regulation introduces a mechanism of mutual recognition of notified electronic identification schemes between Member States.

Clearly, the cross-border transfer of information raises interesting issues regarding the need for a harmonization of rules, processes and safeguards both in Europe and globally. In this sense, the EU is promoting activities in the field of eHealth interoperability and standardization. Specifically, the eHealth Network (eHN) has been created by the Directive 2011/24/EU to achieve coordination, coherence and consistency. It is co-chaired by the European Commission and Austria and it draws up guidelines on how to apply patients' rights in crossborder healthcare. In general, the network aims to enhance interoperability between electronic health systems for continuity of care with guidelines on a minimum/non-exhaustive PS dataset for electronic exchange in accordance with the cross-border directive 2011/24/EU [10]. The new guidelines specify how patients, upon explicit request, can have a summary of their EHR available when visiting another country in the EU. Similar guidelines have been adopted by the eHN in 2014 for the eP minimal dataset [11].

To stimulate the development of generic cross-border eHealth services, 16 Member States received financial support under the Connecting Europe Facility (CEF). CEF is a key EU funding instrument [12] of the Innovation and Networks Executive Agency (INEA), aiming to promote growth, jobs and competitiveness through targeted infrastructure investment at European level, supporting interconnected trans-European networks in the fields of transport, energy and digital services. CEF is the financial framework under which the eHealth Digital Service Infrastructure (eHDSI or eHealth DSI) initiative is carried out, and the eHN guidelines are the reference for the electronic exchange of health data adopted by eHDSI. The eHDSI is the initial deployment and operation of services for cross-border health data exchange [13], meant as a move from the epSOS conceptual framework to its deployment phase. Whenever real patient data are exchanged, the $\mathrm{NCPeH}$ must be in conformity with the agreed principles as adopted by the eHN.

In 2015, a "Joint Action to Support the eHealth Network" (JASeHN) was launched to support eHN. In JASeHN public health authorities and other stakeholders of all Member States are currently collaborating and developing recommendations for the eHN. An important document produced by JASeHN and adopted by eHN in 2017 is the "Agreement between National Authorities or National Organisations responsible for National Contact Points for eHealth on the Criteria required for the participation in Cross-Border eHealth Information Service" [14]. According to this agreement, cross-border exchange of health data can only happen when the Member States involved enter into an agreement for this specific purpose, legally based on the national law of the respective Member State. In particular, the agreement determines the following important points:

- The mechanisms of identification of patients, health professionals and healthcare providers have to follow the eIDAS Regulation.

- The country of treatment shall ensure that only health professionals authorized according to its national law may have access to patients' data concerning health.

- Each Contracting Party shall designate one $\mathrm{NCPeH}$ to act as a single communication gateway with the $\mathrm{NCPeH}$ designated by other Contracting Parties.

- Each country is responsible for the accuracy and integrity of semantic processing.

- Each Contracting Party shall ensure the compliance of its NCPeH with the principles of data protection by design and by default, the requirements for confidentiality, integrity, authenticity, availability, non-repudiation, encryption, logs, audit trails, and other means of data security and control measures in compliance with Regulation 2014/910/EU and Regulation 2016/679/EU.

Table 1 summarizes the main legal and organizational requirements previously described and the main actual barriers to their applications.

\subsection{Ethical aspects}

In order to understand what ethical principles have already been identified and discussed in the context of eHealth and cross-border health data exchange, a comprehensive analysis of the recent literature and European Regulations has been conducted [15]. In Fig. 1, the main findings are aggregated, identifying a minimum dataset of ethical principles for cross-border applications. Table 2 translates the ethical principles into pragmatic actions explaining how the abstract ethical principles may be included and implemented into cross-border solutions designed according to the "ethics-by-design" principle [15].

Summarizing, Table 3 depicts the main ethical requirements previously described and the respective challenges.

\section{The European infrastructure for cross-border health data exchange}

The eHN released the "Guideline on an Organisational Framework for eHealth National Contact Point" [16]. The main architectural element of this framework is the $\mathrm{NCPeH}$, which constitutes the country's communication gateway providing the interface (not only technical) 


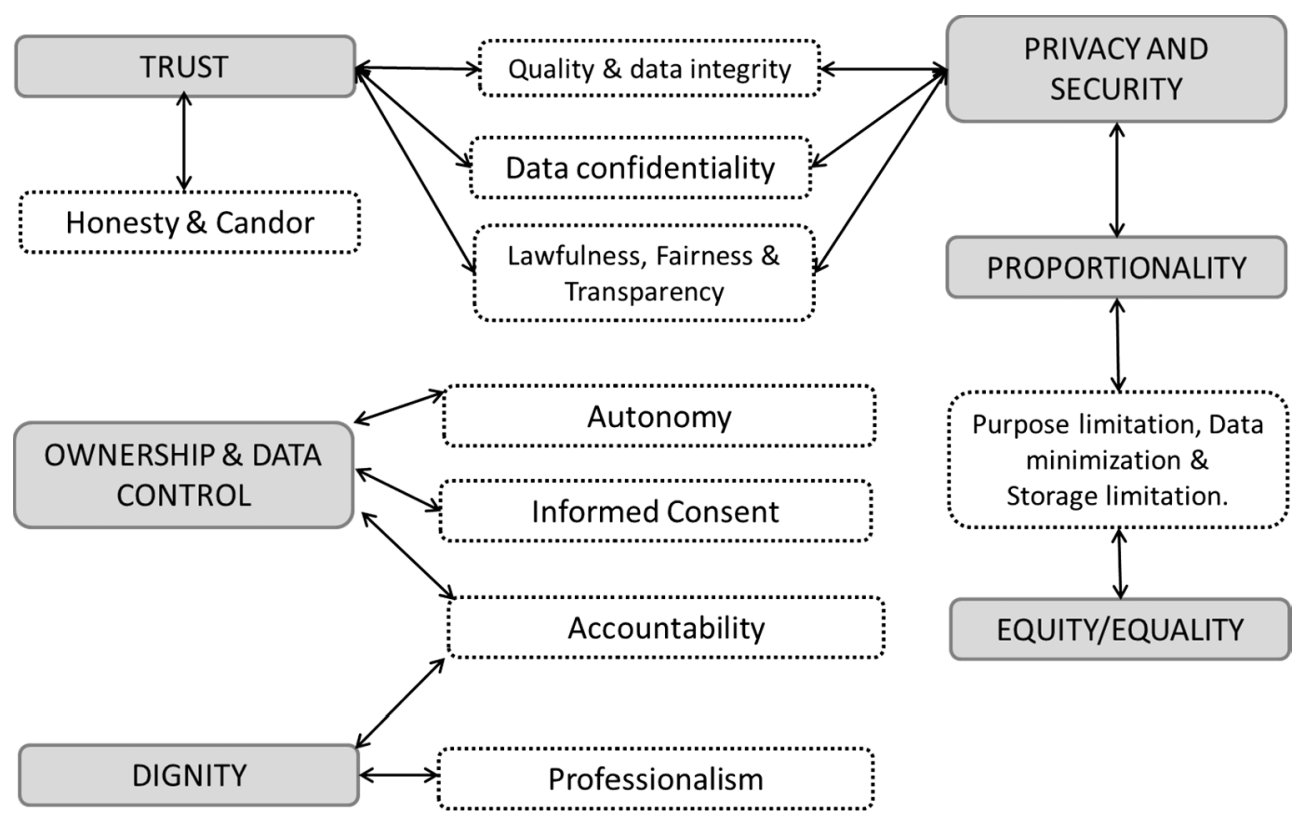

Fig. 1. Aggregation of literature findings about ethical principles related to eHealth and cross-border health data exchange (updated from [15]).

between the National Infrastructure (NI) and the EU network of other Member States' $\mathrm{NCPeH}$, as well as with the central EU services. The $\mathrm{NCPeH}$ must be recognizable both in the EU domain (with the NCPeH of other countries) and in the national domain, acting as the main interface between the two. Every $\mathrm{NCPeH}$ can work in two different scenarios, when a patient is travelling abroad for any reason (holiday, study, work relocation, etc.):

- Country-A: It is the Country of Affiliation, i.e., the country which holds information about a patient, where the patient can be univocally identified and where his/her data may be accessed;

- Country-B: It is the Country of Treatment, i.e., the country where cross-border healthcare is provided, when the patient is seeking care abroad.

Different EU Member States will deploy their NCPeH in different moments, based on the eHDSI NCPeH service deployment plan [17].

In Italy, the implementation of this infrastructure was carried out through a project of the Italian Ministry of Health, the Digital Agency (AgID - Agenzia per l'Italia Digitale) and three pilot regions (Lombardy, Veneto and Emilia-Romagna). This CEF-funded project is called "Deployment of Generic Cross Border eHealth Services in Italy" (call: eHealth 2015 CEF-TC 2015-2) [18] started on January the 1st 2017, and ends in 2020. The project includes the following activities:

- Governance and management;

- NCPeH architectural design;

- NCPeH development and deployment;

- Communication and training strategy.

In the cases in which Italy acts as the Country of Affiliation (Country-A) of an Italian citizen abroad, the Italian $\mathrm{NCPeH}$ receives the request to retrieve the PS or eP. The Italian National Infrastructure is used to verify the citizen's personal data and the presence of the consent to share clinical data abroad and then it interconnects the Italian $\mathrm{NCPeH}$ to regional systems in order to retrieve the respective documents (PS and eP).

On the other hand, when Italy is acting as the Country of Treatment (Country-B) for a foreign patient, the Italian $\mathrm{NCPeH}$ will provide a dedicated portal which will forward the request to the $\mathrm{NCPeH}$ of the Country of Affiliation (Country A), receive the PS or eP, and provide the medical practitioner or pharmacists the tools for their visualization.

One of the key elements of this eHealth services platform is that PS and $\mathrm{eP}$ are shown to the doctor or pharmacist in their language. The original documents provided by Country A are transformed, transcoded and translated. The semantic components of the NCPeH have in fact the

Table 2

Ethical principles and suggested actions.

\begin{tabular}{|c|c|}
\hline Ethical Principles & Suggested actions \\
\hline Trust & $\begin{array}{l}\text { - Include appropriate data quality mechanisms and integrity checks. } \\
\text { - Data needs to be collected in a standardised way, so that it can be comparable and usable. } \\
\text { - Patients must be informed about policies and practices regarding the data exchange that should be provided in a clear and understandable form. } \\
\text { - The point of care has to inform the patients regarding potential breaches of data security. }\end{array}$ \\
\hline Privacy \& Security & $\begin{array}{l}\text { - Perform a threat/risk analysis to identify the principle potential information security weaknesses and related remedies. } \\
\text { - Prepare an information sheet with details about the security measures. }\end{array}$ \\
\hline Proportionality & $\begin{array}{l}\text { The data sharing mechanisms should guarantee that the data are not stored longer than necessary in the recipient country and the information is } \\
\text { unobstructed when there is an urgent need to obtain data, particularly to prevent loss of life. }\end{array}$ \\
\hline Ownership \& Data control & $\begin{array}{l}\text { The patients have to be informed about the processing of the personal data and they must authorise data manipulation (e.g., provide authorisation } \\
\text { for cross-border health data sharing). }\end{array}$ \\
\hline Equity/Equality & $\begin{array}{l}\text { Cross-border solutions should contribute to equality in healthcare and they should be usable in every EU member country and by each citizen of the } \\
\text { EU. }\end{array}$ \\
\hline Dignity & $\begin{array}{l}\text { - Cross-border solutions should be designed without ignoring the human aspects and patient rights. The patient is at the centre of the healthcare } \\
\text { processes. }\end{array}$ \\
\hline
\end{tabular}


Table 3

Ethical requirements and barriers.

\begin{tabular}{|c|c|}
\hline Main Ethical Requirements & Barriers \\
\hline High level of trust and security & $\begin{array}{l}\text { European Level: } \\
\text { - Lack of harmonization of rules, processes and safeguards. } \\
\text { - Lack of official and shared security practices. } \\
\text { National/Local level: } \\
\text { - Lack of management commitment at local level of point of care. } \\
\text { - Lack of the budget to address security aspects by healthcare } \\
\text { organizations. } \\
\text { - Lack of specific security policies. }\end{array}$ \\
\hline $\begin{array}{l}\text { Respect patients' rights: Right to access data, the right to erase and correct data and the right to know } \\
\text { who accessed data. }\end{array}$ & $\begin{array}{l}\text { - NCPeH are still in early stages. } \\
\text { - Lack of official harmonization of rules. }\end{array}$ \\
\hline
\end{tabular}

purpose to carry out the transformations on the documents to be sent and the translations of the received documents, safeguarding the semantic content and the medical-legal validity. Specifically, the information from Country A is shown to the healthcare professional in a structured way, i.e. in structured modular data groups sorted under the correct nesting headlines; each of those data groups contains related items of information with a unified meaning of fields (e.g. field 'Active Problems' is properly translated to Country B) [19]. A specific connector component of the Italian $\mathrm{NCPeH}$ transforms the Italian Synthetic Health Profile (the PS contained into the Italian EHR) into a PS syntactically compatible with European specifications. Similarly, it transforms the Italian dematerialized recipe into an eP consistent with the European specifications [19].

The NCPeH itself currently will remain in pre-production phase until a proper audit and test from the eHN can occur: after these steps, the NCPeH will enter the production phase and will be fully activated.

The IPSE project ("European and National Interoperability solutions for the Electronic Healthcare Record: Patient Summary and ePrescription components"/Interoperabilità europea e nazionale delle soluzioni di fascicolo sanitario elettronico: componenti Patient Summary e ePrescription) is relevant with the creation of the Italian National Infrastructure and it influences the deployment of the NCPeH [20]. IPSE was carried out by the Italian Ministry of Health and 10 Italian Regions between 2010 and 2012, and it started the definition of a National Interoperability infrastructure based on the epSOS concepts. In particular, together with the NCPeH node, IPSE defined an Interregional Contact Node (ICN), coordinating a set of Regional Contact Nodes (RCN) to be implemented by each Region, with a similar federated model with respect to the one defined by the eHN between EU Member States. Fig. 2 illustrates the IPSE infrastructure.

Moreover, IPSE defined reusable epSOS building blocks [21], in particular:

- The legal framework.

- End-2-end security, in particular:

o VPN based on SHA-2 certificates.

o Encrypted and signed messages.

o No data storage at NCP level.

o Audit trail for every transaction.

- Adoption of IHE (Integrating the Healthcare Enterprise) profiles.

- Semantic Interoperability.

\section{The Italian eHealth data infrastructure}

The Italian healthcare system is based on a national framework called National Healthcare Information System (Nuovo Sistema Informativo Sanitario - NSIS) that defines, at a national level, the specification for a unified EHR. However, as the Italian healthcare system is organized in a federation of Regions, consequently all the implementations and deployment plans, as well as the data collection and management need to be developed at a regional level. The Agency in charge of Italy's Digitalization (AgID), in cooperation with the Ministry of Health and the Ministry of Economy and Finance, designed the National Interoperability Infrastructure (Infrastruttura Nazionale per l'Interoperabilità - INI) to exchange information among the different Regions and speed-up the deployment of EHR for Regions. The Italian EHR is called Fascicolo Sanitario Elettronico (FSE), and it will be used by each Region, following national rules within the technological framework of the Healthcare Insurance card - Tessera Sanitaria (TS System). The FSE is activated with the signature of the informed consent which explains what is the FSE, what its activation means, the purposes and the authorized operator of the Italian healthcare system that can consult and update FSE content. The FSE can be activated by the general practioner/pediatrician or by using a dedicated online portal with proper authentications (with the Public System for Digital Identity SPID). Access to FSE is regulated by procedures established by national law and applied autonomously by the Regions. Currently, the FSE is not activated and implemented in all Italian regions [22].

The National regulation about FSE (Decree of the President of the Council of Ministers - dPCM n. 178/2015) [23] defines all healthcare data about the patient contained into FSE: laboratory records, therapies, medical history (documents that are part of the FSE), provisions for the protection of the privacy of the patients, the governance system, data collection process, the data encoding systems. Furthermore, it contains an essential version of the FSE which is called Profilo Sanitario Sintetico or Patient Summary, which includes the same information of the PS defined in epSOS and by the eHDSI. The PS is managed by the general practitioners or paediatricians, who have the responsibility to update its content according to administrative and medical information. In particular, the FSE Minimum Dataset and the relevant documents included are listed in Table 4.

The complete picture for the Italian Health National Infrastructure, including all the components needed for cross-border health data exchange (e.g., NCPeH) is presented in Fig. 3, in which four levels are differentiated:

1) The European level: the NCPeH will be instantiated within 2020, allowing Italy to act both as Country-A and Country-B in the data exchange process defined in epSOS. This node will follow the standards defined by eHDSI and it will become operational after appropriate testing and auditing processes $(\mathrm{NCPeH})$.

2) The National level: the NCPeH node will contact the INI to route the requests in the relevant Region. INI will orchestrate the data exchange with all the Regions. In the second quarter of 2018, 17 Regions are declared active in trying to implement their own FSE system, while 11 of them are already connected with the INI network [22].

3) The Regional level: this involves the specific Regional FSE system. There are two different models for the Regional level. In the first model, the documents index and the documents themselves are in the same (Regional) system (saved in structured or unstructured format: HL7 CDA v2 or PDF). In the second model, the Regional 


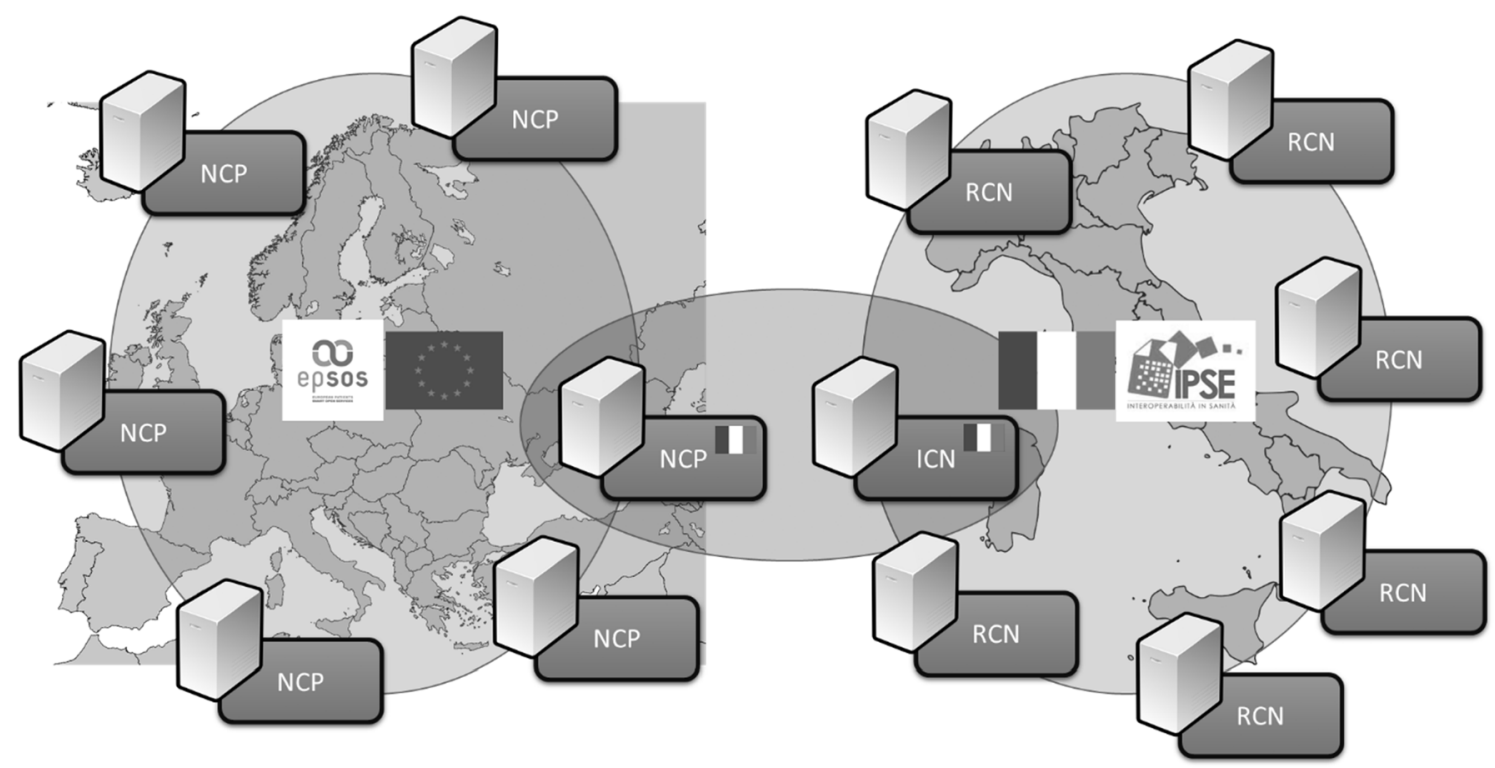

Fig. 2. The IPSE infrastructure based on epSOS (new nodes were defined, in particular the Interregional Contact Node (ICN) and Regional Contact Nodes (RCN)) (). Adapted from [20]

system is composed only by the clinical documents' index (including metadata), while all the documents are shared between the local Document Repositories in the regions, located in hospitals, GPs offices, etc.

4) The Local level: we can find all the end-users of the system, being both Italian citizens accessing eHealth services or Healthcare Professionals dealing with patients.

The INI node, which is in charge for the exchange of any clinical document between Regions, has a defined set of processes to be managed: patient identification, consent management, document search, document retrieval, meta-data management (creation, update, deletion), documents' reference retrieval, index transfer (to another Region). This node relies on the National Health Card System (Tessera Sanitaria - TS System) for the identity information of the patient.

In Italy, the PS is a subset of information contained in the FSE, and it is an actual clinical document exchanged like all the other clinical documents in Italy. It must be prepared by the treating GP of the patient and it must include: patient and GP IDs, list of diseases, diagnosis, allergies, pharmacological therapies, chronic conditions and all the information needed to guarantee the patient care. In case of change of GP, the new GP will have the responsibility to update the PS, with his ID and updated information about the patient.

The eP (Fig. 3) follows two separate flows (regulated by the Ministry
Decree of the 2nd November 2011), in line with the European system:

- An eP consists of a unique number (Numero di Ricetta Elettronica NRE), issued by SAC (Sistema di Accoglienza Centrale - a subsystem of the TS System), which allows doctors, pharmacies, and public or private healthcare entities to exchange information about the prescription in real time. GPs have the possibility to issue a paper version of the prescription in case of system malfunctions. In some cases, there is a Regional node releasing the NRE (called SAR), which interacts with the SAC to make sure that there is alignment between the two systems.

- The eD, where the drug is released, and the information is forwarded to the National Health Smart Card System, again by the NRE. Using the NRE and the fiscal code, the dispenser forwards the communication to the SAC/SAR to recover the treatment or the drug that the patient needs, and when the eD is closed, he/she sends the financial information back to the SAC/SAR with the eventual costs paid by the patient.

Table 5 consolidates the legal, organizational and ethical requirements identified in Tables 1 and 2. For each of them describes the solutions applied in the Italian context at each level of Fig. 3.

Table 4

FSE documents according to dPCM n. 178/2015, differentiating the minimum data set and the extended data set [23]

\begin{tabular}{|c|c|c|}
\hline \multirow{2}{*}{$\begin{array}{l}\text { Minimum Dataset } \\
\text { Identification and administrative data of the patient }\end{array}$} & \multicolumn{2}{|r|}{ Other Documents } \\
\hline & Prescriptions & Hospital care \\
\hline Medical reports & $\begin{array}{l}\text { Bookings for hospitalization or specialist's } \\
\text { visits }\end{array}$ & Medical certificates \\
\hline Emergency reports & Medical records & Patient's personal notebook \\
\hline Discharge letters & Health checks & Continuity of care \\
\hline Patient summary & Home care & Self-certifications \\
\hline Pharmaceutical dossier & Diagnosis and treatment plans & Participating in clinical trials \\
\hline \multirow[t]{5}{*}{ Choice regarding the donation of organs and tissue } & Semi-residential care & Co-payment Exemptions \\
\hline & Dispensing medications & Prosthetic assistance \\
\hline & Vaccinations & Data to support the activities of tele-monitoring \\
\hline & Outpatient care & $\begin{array}{l}\text { Data to support the activities of the integrated management of diagnostic } \\
\text { and therapeutic }\end{array}$ \\
\hline & Emergency care & Other relevant documents \\
\hline
\end{tabular}




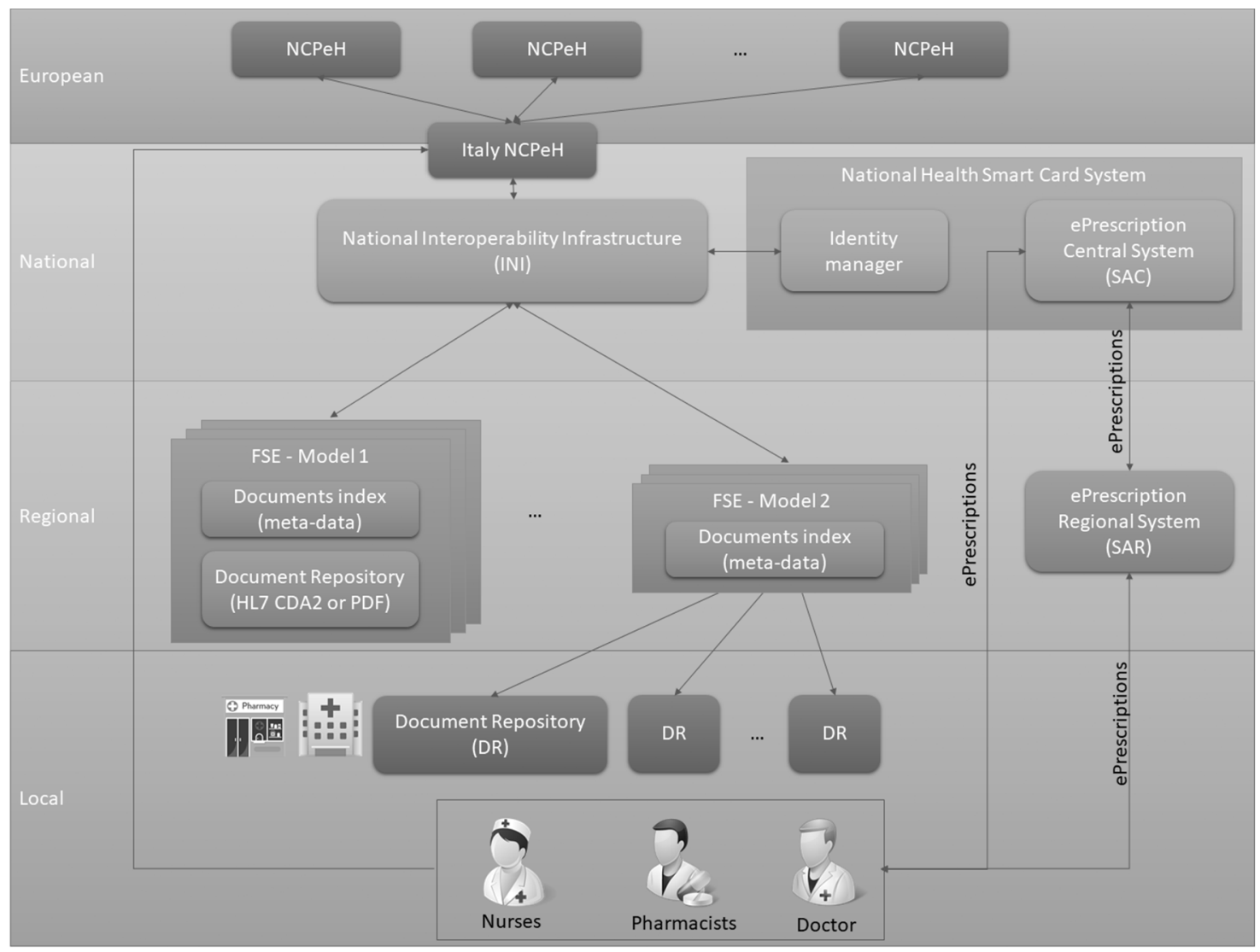

Fig. 3. Italian eHealth data Infrastructure.

\section{Reinforcing security of the EU eHealth data infrastructure through the KONFIDO toolset}

KONFIDO is an EU-funded research project aiming to develop a holistic paradigm for secure cross-border health data exchange. It builds its solution upon existing/evolving European frameworks, such as OpenNCP [24-26], the open-source NCP reference software implementation part of project epSOS, and eIDAS (electronic IDentification, Authentication and trust Services). In epSOS, the security of communications is ensured by employing cryptography and appropriate protocols. However, the security of communicating parties is enforced via legal agreements and technical means; it is instead pretended by legally binding agreement. Furthermore, epSOS does not offer any protection against the propagation of cyber-attacks. Therefore, attacks which succeed in compromising a NI can exploit the NCP to propagate to other countries. This means that, due to this chain of trust between the NCPs, if one NCP states that someone is authenticated, this will be accepted by the NCPs of other countries. More specifically, the PS is retrieved in plain-text format. This means that a vulnerability of NCP can generate a data breach on the OpenNCP processes.

KONFIDO developed a toolset which can be used to overcome the identified vulnerabilities by deploying a set of functionalities to guarantee, for example, that the medical data will be never exposed as plaintext in a non-secure area. KONFIDO improves OpenNCP security with a set of security tools organized in an easily pluggable architecture [26]. Specifically, the toolset offered by KONFIDO includes the following tools/services:

- Trusted Execution Environment (TEE): the new security extensions provided by some of the main CPU vendors; focuses on the enhancement of the NCP Connector, the component that translates the
PS. During the PS translation, healthcare data may be exposed to attacks (for example, dumping the memory of the OpenNCP process in charge of document transformation processing). KONFIDO aims to protect the sensitive information with a manipulation of the PS inside the TEE [27].

- Physical Unclonable Function (PUF): PUF-based security solutions that rely on photonic technologies to generate strong keys. PUF is a one-way mathematical transformation that is based on complex and non-reproducible physical mechanisms. Specifically, KONFIDO employs photonic PUFs (p-PUF) to generate random numbers for the creation of cryptography keys) [28,29]; PUF keys are used to establish secure communication links between NCP nodes and/or other KONFIDO modules.

- Homomorphic Encryption (HE) mechanisms: The HE component is used to protect the exchange and the processing of patient data at NI level and in the NCP context [30]. HE is a cryptographic method allowing to execute algorithms directly on encrypted data, without the need of decrypting them, thus without violating data privacy [30]. KONFIDO's p-PUF key-generator device will also be applied to establish the homomorphic encryption keys.

- Customized extensions of Security Information and Event Management (SIEM) solutions [31]: will be able to analyze information and events collected using a holistic approach at the different levels of the monitored system to discover possible ongoing attacks, or anomalous situations.

- Blockchain-based auditing: a set of logging and auditing mechanisms particularly tailored for the healthcare domain [32]; enables to prove that healthcare data have been requested by a legitimate entity and whether they have been provided or not.

- A customized eIDAS implementation; eIDAS-compliant authentication will enable patients to authenticate themselves based on Country A data, while being in Country B and to provide consent in a not 
Table 5

Legal, Organizational and Ethical Requirements and solutions that will be implemented in Italy at European and National/Regional Level.

Main Legal, Organizational \& Ethical Requirements Italian European level solutions Italional/Regional solutions
(Table 1)

Patient Identification

Patient consent management

Healthcare provider identification

Management of healthcare professional authorization/ certification

Enhance interoperability

Accuracy and integrity of semantic processing. ach NCPeh should be compliant with confidentiality, integrity, authenticity, availability, non-repudiation, encryption, logs, audit trails, and other means of data security

High level of trust and security

Respect of patients' rights: Right to access data, the right to erase and correct data and the right to know who accessed data.
The Italian infrastructure will provide an eIDAS compliant system for Italian patients. It will recover foreign patients' identities from the other countries eIDAS systems.

The same national system is used also at international level.

The same interregional system will be used at international level.

In the international context, when Italy is operating as Country B, they will authenticate with SPID anyway, but consulted directly by the OpenNCP node instead of the usual regional FSE.

Interoperability between European countries will be ensured trough the implementation of OpenNCP. The "Deployment of Generic Cross Border eHealth Services in Italy" initiative will implement the roadmap within 2020. EU Member States will exchange patient summary and ePrescription only.

This will be ensured by the deployment of the NCPeH.

This will be ensured by the deployment of the NCPeH.

The patient is identified through a national health smartcard system, with SPID identification system.

The consent management process is defined at the National level, despite the fragmentation of the regional healthcare systems. Every region will collect consent for its citizens and it will be provided to the other Regions upon request.

The National smart card system defined clear procedure and roles for the identification and authorization of the healthcare providers.

At the regional level, each healthcare professional will authenticate using the same SPID system used by the patients. They are then authorized at regional level by the healthcare providers.

In Italy, interoperability is defined at the National level and implemented trough the Italian interoperability infrastructure (INI). 11 Regions already adhered to INI and others have plans to join it in the future. Regions will be able to exchange the full FSE.

The IPSE project proposed to test the semantic interoperability building block of epSOS also at National level: even if the language is the same, there are different regional names and definitions which must be harmonized.

The IPSE project claimed that security and auditing mechanisms defined by epSOS will be exploited also at National level.

Adoption of the eHealth Network guidelines in the implementation of the NCPeH.

Definition of the common framework of the National FSE and the INI.

Clear consent management and consent revoke processes are defined at National level and implemented at Regional level. repudiable manner [33].

The EU's recommendations for the last few years [8] have tended to stress the protection of privacy and security of individuals and organizations in the domain of cyberspace. Healthcare is obviously a critical area, because it also deals with individuals and organizations that are more vulnerable than the general public. As a cybersecurity project that targets the security of the field of transnational, European healthcare support systems, KONFIDO aims to reinforce the security measures of OpenNCP $[24,34]$, also by detecting and mitigating its potential misuse, e.g. malicious attacks [35-37]. To first detect and then counter a misuse, monitoring and control actions can be carried out at the level of NCPeH-Country A and NCPeH-Country B [35]. At a systemic level, further controls can also be carried out. For example, the detection and tackling of misuse attempts could be utilised again to identify similar behaviours, using for example machine learning techniques to facilitate the prevention of future attacks. However, this comes at a cost with added load and congestion on the detection and mitigation system, since repeated attack attempts under different guises will create overload in different system components. This means that even with perfect detection and mitigation systems, detection and cybersecurity control schemes themselves will create additional workload and possible congestion, which has to be evaluated, quantified, and for which resources have to be provisioned during normal system operations [35]. Moreover, detection schemes are obviously imperfect and will result in some false alarms. Thus, their evaluation should include the costs of these false alarms, including the additional delays, and possible user dissatisfaction and frustration caused by false alarms.

\subsection{KONFIDO deployment in Italy}

To make sure that KONFIDO has a high and practical impact, its technical solution is going to be tested in three substantial pilots in three distinct European countries (namely, Denmark, Italy, and Spain) [38]. In the Italian context, Italy acts as the Affiliated Country. Besides the above, this section refers to the foundations of the respective validation framework.

\subsection{The Italian scenario: An example}

Anna is from Italy, Lombardy region, and she is on vacation in Spain. Thanks to KONFIDO, Anna knows that in case of problems, any certified healthcare professionals in Barcelona will have access to her PS in a secure way. During the journey, Anna faints and decides to go to the nearest hospital in Barcelona to check her health conditions. The healthcare professional of the hospital (Local Point of care) connects to the Spanish NCPeH and identifies and authenticates Anna and his/ herself using eIDAS. The action of authentication and the request for Anna's PS are all enhanced by KONFIDO. The retrieval request is sent to the Italian $\mathrm{NCPeH}$ that, using the National Interoperability Infrastructure (INI) node of the national architecture of the FSE, performs the following actions: 
- Verifies Anna's personal data and the validity of consent to send clinical data abroad.

- Interconnects to the Lombardy regional systems.

- After consent verification, the regional FSE system retrieves the required clinical documents from a document repository.

- Then, a specific component of the NCPeH, called National Connector, transforms in a TEE the Italian Synthetic Health Profile into a PS syntactically compatible with European specifications.

- Finally, a dedicated portal enables the health professional to visualize the document.

\subsection{The Italian pilot: Scenario implementation}

As the actual Italian National Infrastructure is not available to a research project such as KONFIDO as a test-bed for malicious misuses, unintentional misuses, and actual system attacks, the project will realize a testbed which will support the piloting activities of the developed technologies. This testbed will be composed by a mix of KONFIDO security components and software stubs, i.e. prototype software artifacts implementing the same interfaces of existing components (or those currently under development/testing by the Italian Ministry of Health).

The Italian testbed architecture is illustrated in Fig. 4, and it is composed of:

- A KONFIDO-enhanced OpenNCP deployment, simulating the Italian $\mathrm{NCPeH}$. This node will be responsible for the information exchange between the other KONFIDO testbeds (which will be deployed in Spain and Denmark) and the Italian National Infrastructure.

- A prototype Web portal of OpenNCP for Italian clinicians, who may wish to retrieve a foreign patient's PS and/or eP;

- A stub component which acts as the INI, replicating its processes for the interregional communications and exchange of medical information.

- Two Regional stub components, which KONFIDO enhances, simulating Regional FSE (containing the PS and eP).

Table 6 lists the legal, organizational and ethical requirements identified in Table 5, describing the solutions applied in the Italian pilot of Fig. 4.

While the main goal of KONFIDO is to secure the OpenNCP nodes of Member States, the secondary goal of testing some of the security enhancements also at Regional level can be pursued in parallel, and the rationale for this is provided by the IPSE project, the aim of which is to reuse epSOS building blocks also within the INI.

This testbed will be used for the definition of test use cases and misuse cases, both from external attackers and malicious insiders in the involved systems.

\subsection{Pilot validation}

Pilot validation aims to verify that the KONFIDO solution meets the
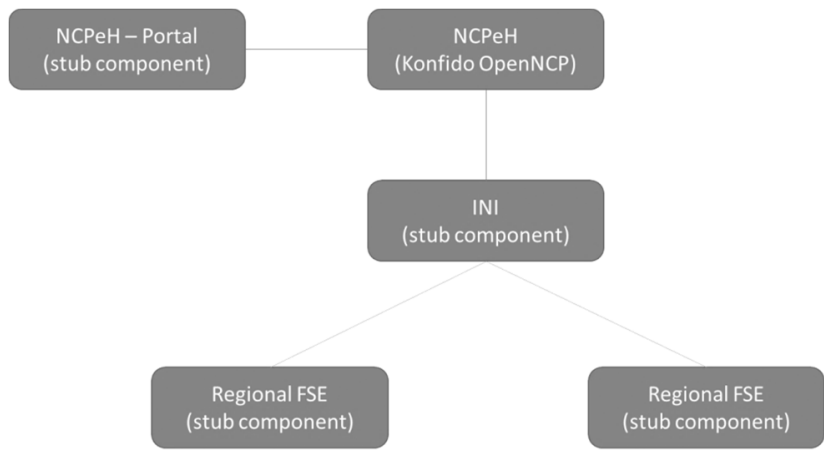

Fig. 4. Italian Test-bed for the KONFIDO Pilot. project objectives, based on data gathered during the pilot phase. The main goal is to prove the following three objectives:

1. The KONFIDO solution actually works.

2. KONFIDO can be integrated with pilot countries' national infrastructure (i.e. EHR systems, etc.).

3. The KONFIDO solution improves the security of exchanging information through OpenNCP.

As part of the validation phase, the activities conducted in the KONFIDO pilots will be reported in detail and the gathered data will also be presented. In order to provide tangible evidence of achieving the three validation objectives, a set of measurable criteria are going to be defined based on the data collected from pilots, as explained in Table 6 . The pilot validation criteria will be based on the following axes:

- User goals as the main result of the KONFIDO user requirements phase [36].

- Misuse cases identified as main threat scenarios.

- Standards and widely accepted best practices.

- The exact validation criteria will be iteratively elaborated during the pilots' phase as they heavily depend on the data collected. However, Table 7 lists some indicative criteria defined as part of the pilot validation planning process.

Lessons learned and challenges will also be elaborated to produce the final validation conclusions and identify opportunities for future work as well as a strategy for the transferability of the KONFIDO outcomes to other EU Member States.

\section{Discussion}

The development of a unified European framework for the exchange of health-related data is a critical problem that needs to be solved, in order to match the regulatory frameworks at the European level and within the EU Member States and because of the sensitive information that is exchanged. Thus, a "privacy by design" and "ethics by design" approach must also be undertaken to generate trust in the end-users and unlock eHealth potentialities and facilitate the framework's adoption across Europe.

The European Commission invested in addressing the issue of crossborder health data exchange with the epSOS project, and with several other initiatives following its proposed model. However, the actual adoption and implementation of this model requires time and effort by EU Member States, in order to define appropriate processes, regulations and develop and deploy technological infrastructures [39]. In this context, the major barriers identified can be summarized as follows [40]:

- Not all EU Member States are aligned with the JASeHN agreement.

- Different consent mechanisms exist among Member States.

- Lack of standard EHR system in Member States.

- Different implementation of EU regulations among Member States.

- Different information workflows among National Infrastructure and healthcare organizations.

- Lack of harmonization in rules, processes, and safeguards.

- NCPeH deployments in Member States are still in early stages.

- Lack of the budget to address security aspects by healthcare organizations.

\section{Conclusion}

In this paper, we described the process that Italy followed for the development of the epSOS model, implementing its own NCPeH in parallel with the development of the National EHR (called FSE). Since the Italian Healthcare System is federated and administered regionally, 
Table 6

Legal, Organizational and Ethical Requirements and solutions that will be implemented in the Italian Pilot.

\begin{tabular}{|c|c|}
\hline Main Legal, Organizational \& Ethical Requirements (Table 1) & Italian pilot solutions \\
\hline Patient identification & Test an enhanced, customized version of the eIDAS system. \\
\hline Patient consent management & $\begin{array}{l}\text { Implement a stub virtual process for consent management in line with the actual } \\
\text { National system. }\end{array}$ \\
\hline Healthcare provider identification & $\begin{array}{l}\text { The identification of healthcare providers is not a main focus for validating KONFIDO } \\
\text { technologies, thus two ad hoc healthcare providers will be preconfigured in the Italian } \\
\text { testbed. }\end{array}$ \\
\hline Management of healthcare professional authorization/certification & $\begin{array}{l}\text { The same stub for digital identity used for the patients will be used also for healthcare } \\
\text { professionals. As for the healthcare providers, they will be pre-configured in the } \\
\text { testbed. }\end{array}$ \\
\hline Enhance interoperability & $\begin{array}{l}\text { Enhanced interoperability will be tested in the pilots using KONFIDO solutions both at } \\
\text { NCP level and regional level. }\end{array}$ \\
\hline Accuracy and integrity of semantic processing & $\begin{array}{l}\text { Leverage on the OpenNCP functionalities (like OpenNCP transformation service) } \\
\text { secured by KONFIDO (leveraging on the trusted executing environment). }\end{array}$ \\
\hline $\begin{array}{l}\text { Each NCPeH should be compliant with confidentiality, integrity, authenticity, } \\
\text { availability, non-repudiation, encryption, logs, audit trails, and other means of data } \\
\text { security }\end{array}$ & $\begin{array}{l}\text { This is one of the main pilot focus, leveraging on the innovations provided by } \\
\text { KONFIDO, both at national and international level, and the added value will be } \\
\text { validated. }\end{array}$ \\
\hline High level of trust and security & $\begin{array}{l}\text { Test the security enhancements proposed by KONFIDO both at National and } \\
\text { International level. }\end{array}$ \\
\hline $\begin{array}{l}\text { Respect of patients' rights: Right to access data, the right to erase and correct data and } \\
\text { the right to know who accessed data. }\end{array}$ & $\begin{array}{l}\text { As patients are defined in KONFIDO as beneficiaries of the innovation, but not as the } \\
\text { main users, the respect of their rights will be considered implicit and will not be tested. }\end{array}$ \\
\hline
\end{tabular}

the implementation of the FSE is itself federated and each Region can deploy its own version. This deployment is an ongoing process, which has a different level of maturity across Regions.

In this scenario of federations of different actors at Regional, National and European level, several threats to security and integrity of personal health data might arise. Thus, the KONFIDO project aspires to demonstrate how innovative technologies may be deployed in realistic scenarios that are compliant with legal and ethical principles. To this end, KONFIDO identified key barriers and facilitators, and implemented a first integrated prototype for secure cross-border eHealth data exchange. The next steps include the development of the second integrated prototype and the validation of the KONFIDO toolset via pilots implemented in Italy, Denmark, and Spain within 2019. In particular, the Italian pilot will implement a test-bed to validate the KONFIDO solution in near real-world conditions and also elaborate on methods to overcome the identified key barriers via a customized eIDAS system and an enhancement of the functionalities of OpenNCP in terms of consent management and security features, as described in detail in Table 6. The outcomes and lessons learnt from the pilot validation will be made publicly available by the end of 2019. Building upon these outcomes, as future work, we will study the complete use of FSE through different Italian Regions (that not only includes PS and eP, but any kind of clinical document) and further test the KONFIDO capabilities with an overall cost/benefit evaluation of misuse and attack detection schemes.

\section{Conflict of interest}

Author declares that there is no conflict of interest.

\section{Acknowledgements}

We would like to acknowledge and thank Ioannis Komnios, the KONFIDO project coordinator, for his contribution during the revision of this manuscript.

\section{Funding}

The research leading to these results has received funding from the European Union's Horizon 2020 research and innovation programme under grant agreement No 727528 (KONFIDO - Secure and Trusted Paradigm for Interoperable eHealth Services).

This paper reflects only the authors' views and the Commission is not liable for any use that may be made of the information contained therein.

\section{Appendix A. Supplementary material}

Supplementary data to this article can be found online at https:// doi.org/10.1016/j.jbi.2019.103183.

Table 7

Preliminary criteria for pilot validation.

\begin{tabular}{|c|c|c|}
\hline Validation Criterion ID & Criterion & Description \\
\hline VC 1 & Percentage of modules successfully integrated & This criterion would highlight the efficacy of the KONFIDO solution components. \\
\hline VC 2 & $\begin{array}{l}\text { Percentage of misuse cases handled better } \\
\text { compared to plain OpenNCP }\end{array}$ & This criterion would depict the contribution of the KONFIDO solution in the targeted misuse cases. \\
\hline VC 3 & Percentage of user goals satisfied & $\begin{array}{l}\text { The finally deployed KONFIDO solution would be compared with the abstract user goals identified in } \\
\text { the overall KONFIDO user requirements process, in order to assess the contribution of the KONFIDO } \\
\text { solution with the overall end-user needs [36]. }\end{array}$ \\
\hline VC 4 & $\begin{array}{l}\text { Technical validation criteria based on security } \\
\text { standards and best practices }\end{array}$ & $\begin{array}{l}\text { A detailed list of relevant validation criteria will be elaborated based on information from widely } \\
\text { accepted IT security standards and best practices (e.g. ISO } 27 \mathrm{k} \text { and NIST ). }\end{array}$ \\
\hline VC 5 & User acceptance criteria & $\begin{array}{l}\text { The KONFIDO Consortium will employ usability testing, questionnaires, interviews, etc., in order to } \\
\text { identify the appropriate solution given the project's overall pilot plan, the available time as well as } \\
\text { financial constraints. }\end{array}$ \\
\hline
\end{tabular}




\section{References}

[1] 2011/24/EU Directive on the application of patients' rights in cross - border healthcare (Cross - Border Directive). https://eur-lex.europa.eu/eli/dir/2011/24/ oj. Accessed on 18 February 2019.

[2] epSOS project website. https://ec.europa.eu/digital-single-market/en/news/crossborder-health-project-epsos-what-has-it-achieved. Accessed on 18 February 2019.

[3] http://www.ehealth-standards.eu/draft-european-standard-17269-the-patientsummary-for-unplanned-cross-border-care-approved/.

[4] http://www.KONFIDO-project.eu/. Accessed on 18 February 2019.

[5] R. Martino, S. D’Antonio, L. Coppolino, L. Romano, Security in Cross - Border Medical Data Interchange: A Technical Analysis and a Discussion of Possible Improvements, Proceedings of the 41st IEEE Annual Computer Software and Applications Conference COMPSAC 2017, (2017).

[6] L. Coppolino, S. D’Antonio, L. Romano, M. Staffa, KONFIDO project: a secure infrastructure increasing interoperability on a systemic level among eHealth services across Europe, Proc. IEEE Int Conf Internet of Things (iThings) and Green Computing and Communications (GreenCom) and Cyber, Physical and Social Computing (CPSCom) and Smart Data (SmartData), 2017, pp. 342-347.

[7] https://ec.europa.eu/health/sites/health/files/cross_border_care/docs/impl directive_presciptions_2012_en.pdf. Accessed on 18 February 2019.

[8] Regulation (EU) 2016/679 of the European Parliament and of the Council of 27 April 2016 on the protection of natural persons with regard to the processing of personal data and on the free movement of such data, and repealing Directive 95/ 46/EC (General Data Protection Regulation) (Text with EEA relevance) ELI: http:// data.europa.eu/eli/reg/2016/679/2016-05-04.

[9] eIDAS website. https://www.eid.as/home/. Accessed on 18 February 2019.

[10] https://ec.europa.eu/health/sites/health/files/ehealth/docs/guidelines_patient_ summary_en.pdf. Accessed on 18 February 2019.

[11] https://ec.europa.eu/health/sites/health/files/ehealth/docs/eprescription_ guidelines_en.pdf. Accessed on 18 February 2019.

[12] https://ec.europa.eu/inea/en/connecting-europe-facility. Accessed on 18 February 2019.

[13] https://ec.europa.eu/cefdigital/wiki/display/EHOPERATIONS/eHDSI + Mission. Accessed on 18 February 2019.

[14] https://ec.europa.eu/health/sites/health/files/ehealth/docs/ev_20170509_co06_ en.pdf. Accessed on 18 February 2019.

[15] G. Faiella, Building an Ethical Framework for Cross-Border Applications: The KONFIDO Project, in: E. Gelenbe (Ed.), Security in Computer and Information Sciences. Euro-CYBERSEC 2018. Communications in Computer and Information Science, Springer, Cham, 2018, , https://doi.org/10.1007/978-3-319-95189-8_4.

[16] https://ec.europa.eu/health/sites/health/files/ehealth/docs/ev_20151123_co01_ en.pdf. Accessed on 18 February 2019.

[17] https://ec.europa.eu/cefdigital/wiki/download/attachments/35210488/eHDSI ServiceCatalogue-ServiceDelivery-OveralDeployment-Plan V2.8 20180621.pdf? version $=1 \&$ modificationDate $=1530600169719 \& \mathrm{api}=\mathrm{v} 2$. Accessed on 18 February 2019.

[18] https://ec.europa.eu/inea/sites/inea/files/cef-tc-2015-2.pdf. Accessed on 18 February 2019.

[19] https://www.fascicolosanitario.gov.it/progettazione-realizzazione-e-collaudo-delNCPeH-e-dei-servizi-transfrontalieri.

[20] http://www.arit.it/aree-tematiche/sanita-elettronica/86-il-progetto-ipse.html. Accessed on 18 February 2019.

[21] http://www.promisalute.it/upload/mattone/documentiallegati/epSOS_13660_629. pdf. Accessed on 18 February 2019

[22] https://www.fascicolosanitario.gov.it. Accessed on 18 February 2019.

[23] Decree of the President of the Council of Ministers - dPCM n. 178/2015.

[24] M. Fonseca, K. Karkaletsis, I. Cruz, A. Berler, I. Oliveira, OpenNCP: a novel framework to foster cross-border e-health services, Stud. Health Technol. Inform. 210 (2015) 617-621, https://doi.org/10.3233/978-1-61499-512-8-617.

[25] M. Staffa, L. Coppolino, L. Sgaglione, E. Gelenbe, I. Komnios, E. Grivas, O.S.L. Castaldo, KONFIDO: An OpenNCP-based secure E health data exchange system, in: E. Gelenbe, P. Campegiani, T. Czachorski, S. Katsikas, I. Komnios, L. Romano, D. Tzovaras (Eds.), Recent Cybersecurity Research in Europe:
Proceedings of the 2018 ISCIS Security Workshop, Imperial College London. Lecture Notes CCIS No. 821, Springer, Verlag, 2018.

[26] M. Staffa, S. Sgaglione, G. Mazzeo, L. Coppolino, S. D'Antonio, L. Romano, E. Gelenbe, O. Stan, S. Carpov, E. Grivas, P. Campegiani, L. Castaldo, K. Votis, V. Koutkias, I. Komnios, An OpenNCP-based solution for secure eHealth data exchange, J. Netw. Comput. Appl. 116 (15) (2018) 65-85, https://doi.org/10.1016/j. jnca.2018.05.012.

[27] L. Coppolino, S. D’Antonio, G. Mazzeo, L. Romano, L. Sgaglione, Exploiting New CPU Extensions for Secure Exchange of eHealth Data at the EU Level, 14th European Dependable Computing Conference (EDCC2018), 2018, https://doi.org/ 10.1109/EDCC.2018.00015.

[28] M. Akriotou, C. Mesaritakis, E. Grivas, C. Chaintoutis, A. Fragkos, D. Syvridis, Random number generation from a secure photonic physical unclonable hardware module, in: E. Gelenbe, P. Campegiani, T. Czachorski, S. Katsikas, I. Komnios, L. Romano, D. Tzovaras (Eds.), Recent Cybersecurity Research in Europe: Proceedings of the 2018 ISCIS Security Workshop, Imperial College London. Lecture Notes CCIS No. 821, Springer Verlag, 2018.

[29] C. Mesaritakis, M. Akriotou, A. Kapsalis, E. Grivas, C. Chaintoutis, T. Nikas, D. Syvridis, Physical Unclonable function based on a multi-mode optical waveguide, Sci. Rep. 8 (2018) 9653, https://doi.org/10.1038/s41598-018-28008-6.

[30] R.A. Hallman, et al., Building Applications with Homomorphic Encryption, Proceedings of the 2018 ACM SIGSAC Conference on Computer and Communications Security, ACM, 2018.

[31] L. Coppolino, S. D’Antonio, L. Romano, L. Sgaglione, M. Staffa, Addressing Security Issues in the eHeatlh Domain Relying on SIEM Solutions, Proceedings of the 41st IEEE Annual Computer Software and Applications Conference COMPSAC, (2017).

[32] A. Theodouli, S. Arakliotis, K. Moschou, K. Votis, D. Tzovaras, On the Design of a Blockchain-Based System to Facilitate Healthcare Data Sharing, 2018 17th IEEE International Conference On Trust, Security And Privacy In Computing And Communications/ 12th IEEE International Conference On Big Data Science And Engineering (TrustCom/BigDataSE), 2018, pp. 1374-1379.

[33] L. Castaldo, V. Cinque, Blockchain based logging for the cross-border exchange of Ehealth data in Europe, in: E. Gelenbe (Ed.), Euro-CYBERSEC 2018. CCIS, Springer, Cham, 2018, pp. 46-56.

[34] M. Staffa, L. Coppolino, L. Sgaglione, E. Gelenbe, I. Komnios, E. Grivas, O. Stan, L. Castaldo. (2018). KONFIDO: An OpenNCP-Based Secure eHealth Data Exchange System. Euro-CYBERSEC2018: 11-27, Springer Lecture Notes Vol. CCIS 821, Springer Verlag, Berlin, https://link.springer.com/chapter/10.1007\%2F978-3-319. 95189-8_2.

[35] E. Gelenbe, P. Campegiani, T. Czachórski, S. K Katsikas, I. Komnios, L. Romano and D.Tzovaras. Security in Computer and Information Sciences. (2018) First International ISCIS Security Workshop 2018, Euro-CYBERSEC 2018, London, UK, February 26-27, 2018, Revised Selected Papers, Lecture Notes Vol. CCIS 821, Springer Verlag, Berlin, 2018, Open Access, https://link.springer.com/content/pdf/ 10.1007\%2F978-3-319-95189-8.pdf.

[36] P. Natsiavas, J. Rasmussen, M. Voss-Knude, K. Votis, L. Coppolino, P. Campegiani, I. Cano, D. Marí, G. Faiella, F. Clemente, M. Nalin, E. Grivas, O. Stan, E. Gelenbe, J. Dumortier, J. Petersen, D. Tzovaras, L. Romano, I. Komnios, V. Koutkias, Comprehensive user requirements engineering methodology for secure and interoperable health data exchange, BMC Med. Inform. Decision Making 18 (1) (2018) 85, https://doi.org/10.1186/s12911-018-0664-0.

[37] J. Rasmussen, P. Natsiavas, K. Votis, et al. (2017). Gap analysis for information security in interoperable solutions at a systemic level: the KONFIDO approach. Precision Medicine Powered by pHealth and Connected Health, vol. 66. Singapore: Springer; 2017, IFMBE Proceedings. p. 75-9. https://doi.org/10.1007/978-981-107419-6_13.

[38] KONFIDO concept paper. https://konfido-project.eu/system/files/private/konfido/ konfido conceptpaper.pdf. Accessed on 18 February 2019.

[39] F. Pecoraro, D. Luzi, M. Cesarelli, F. Clemente, A methodology of healthcare quality measurement: a case study, J. Phys.: Conf Ser. 588 (2015) 1-5.

[40] P. Natsiavas, Identification of Barriers and Facilitators for eHealth Acceptance: The KONFIDO Study, in: N. Maglaveras, I. Chouvarda, P. de Carvalho (Eds.), Precision Medicine Powered by pHealth and Connected Health. IFMBE Proceedings, Springer, Singapore, 2018, , https://doi.org/10.1007/978-981-10-7419-6_14. 